\title{
Youth's responsible sexuality and capability approach: a conceptual framework
}

\begin{abstract}
In this paper we propose a conceptual framework of youth's responsible sexuality through Sen's Capability approach. Its objects are to define the concept of youth's responsible sexuality, to describe in which analytical framework we use the Capability Approach to conceptualize the youth's responsible sexuality. Precedent works were used to do so and we propose a 3 by 5 table as frame. Horizontaly, we have the social structure: individual level, household level and environmental level. Verticaly we do have resources level, conversion factors level, capabilities level, preferential choices level and accomplisment level.
\end{abstract}

Keywords: youth's responsible sexuality, sexual and reproductive health, capability approach
Volume 6 Issue 3 - 2017

\section{Lalla Fatouma Traore, Samba Ibrahim Diop, Sory Ibrahim Diawara}

Department of Public Health and Specialties, University of Science, Mali

Correspondence: Lalla Fatouma Traore, Department of Public Health and Specialties, University of Science, Techniques and

Technologies of Bamako, Mali, Email leilafatouma.Ift@gmail.com

Received: July 12, 2017 | Published: September 07, 2017

\section{Introduction}

Originally conceived in the 1990s as an approach to economic wellbeing, since the 2000s, the Capability Approach has been a topic of discussion among theorists, politicians, philosophers and people Social sciences including those with a particular interest in human health. ${ }^{1,2}$ Indeed, it served as a framework for analysis and/or exploration for the well-being $;{ }^{3}$ to gender inequality; ${ }^{4,5}$ to Self-help $;{ }^{6}$ to poverty $;{ }^{7}$ and to disability. ${ }^{8}$ The focus on the Capability Approach was due to the fact that it revolutionized the way development was viewed and initiated the concept of "human development" in 1990. ${ }^{9}$ The innovation was to put individuals at the center of development and to consider other dimensions other than economic growth to assess development. From Mahbub ul Haq's ${ }^{10}$ reflections on human development, we note that "The objective of development is to create an enabling environment for people to live a long, healthy and creative life." This quality of life, which is necessary for the advent of development, cannot be achieved without the well-being of individuals and good sexual and reproductive health. This unequivocally establishes the linkage with population and development issues that have been brought to the attention of the international community at various international meetings on these issues, particularly the International Conference on Population and Development in Cairo in 1994. Hence our interest in using the Capability Approach to analyze the sexual and reproductive health of young people and to propose a conceptual framework of responsible sexuality among young people. The rationale behind the choice of exploring the sexual and reproductive health of young people in the light of the Capability Approach is linked to the possibility of being able to identify their specificity, their characteristic in relation to their ability to choose the mode of sexuality they want in spite of socio-cultural norms and contribute to the improvement of the services offered to them for a better quality of life. Without being a perfect and unique tool, we thought that the Capabilities Approach (preferential choice) would be a fairly global tool of analysis of the sexuality of young people to define the content of responsible sexuality. In this document, we will first give content to the concept of sexuality responsible for young people. Then we will establish the framework of analysis with the Capability Approach, taking into account the environment in which they live, the resources available to them, their own individual capacities and specificities and their aspirations in terms of life. This part describes the structuring of the elements identified above and the factors of variation identified by Sen itself. Finally, we propose a conceptual framework of responsible sexuality.

\section{Definition of responsible sexuality}

Sexuality has always occupied a central place in the lives of individuals and is therefore of interest to all those involved in promoting a better quality of life. From this point of view, research on human sexuality involves many researchers specialized in various fields (psychology, sociology, anthropology, epidemiology, public health, education, economics, etc.) and who often exchange for better understanding of the subject. Public health, which is our field of intervention, is by its very nature a social and political concept which not only deals with the disease but also with improving the quality of life of the population. As a result, her interest in sexuality has increased significantly with population and development issues that have come to the attention of the international community at various international meetings on these issues. Indeed, in 1994, at the United Nations International Conference on Population and Development in Cairo, ${ }^{11}$ the International community, with 179 member countries committed to making sexual and reproductive health effective for all age groups by 2015 . Five years later, after a review of progress in the implementation of the Program in Cairo, the commitment extended to health, sexual and reproductive rights. ${ }^{12,13}$ Under the auspices of the United Nations General Assembly, the African continent, like other regions and through the African Union, is committed to implementing these resolutions on sexual and reproductive rights while simultaneously contextualizing them and putting them in various reference documents, the best known of which is the Maputo protocol with its action plan. ${ }^{14,15}$ The operationalization of the strategies advocated for the implementation of the Plan of Action on Sexual and Reproductive Health is common to all African Union countries. Assessing the outcomes of this operationalization in different socioeconomic and cultural contexts and the requirements for effective and efficient interventions to improve the sexual and reproductive health of young people ${ }^{16}$ require that we clearly define our vision of responsible sexuality responsible for young people. Strategies against STIs, including HIV/AIDS in favor of young people, refer to the 
following three rules: abstinence, couple loyalty and condom use. However, worldwide statistics on the age of onset of sexual activity, adolescent birth rates, new cases of STIs and HIV, and cases of sexual multi-sex partnership, reveal the limits of these measures. Thus, taking into account the parameters related to young people in relation to the life they would like to carry out and which they would consider to be rewarding, we propose as content of the concept of responsible sexuality the adoption of three essential points encompassing the three areas of prevention: Primary, secondary and tertiary with regard to the consequences that could result from an early sexuality (Box 1). This content does not claim to exclude other measures that have been tried in high impact interventions on young people. It is merely justified in the context of the answer to the question of the achievement to be reached by the young people in the Capability Approach with regard to the responsible sexuality.

\section{Analytical framework}

The framework of analysis that we are going to describe did not have the same objectives as the work of Nussbaum ${ }^{4-17}$ and the team of Pavia. ${ }^{7}$ In order to examine the responsible sexuality for young people through the Capability Approach, while taking account of these works, we decided to stick to a logical structuring between the definition of the Capability Approach, the factors of variation in the benefit that an individual can derive from the use of the resources made available to him or her and the fundamental principles of right and freedom with regard to sexual, reproductive health and rights. Taking into account the definition of capability, ${ }^{18}$ we note that there are three horizontally social structure levels that influence each other in the fulfillment of a capability which, as far as this article is concerned is the responsible sexuality. These three levels are: individual, household and environmental. We have admitted as the starting point the allocations at these different levels, the availability or absence of which, will condition the opportunities offered to young people in relation to education, access to reproductive health products and services and Social Protection. Second, we considered the conversion factors that are all the individual, familial and sociocultural characteristics that can enable young people to use the resources available to them at the social levels to have the capability necessary for the realization of sexuality responsible. This capability, in our context, is the set of knowledge, attitudes and practices that are essential to make the appropriate preferential choice according to what young people consider to be rewarding and/or their life project. The same socio-cultural context does not necessarily mean the same aspirations or expectations for all young people living in this context. Thus the part of subjectivity existing between a capability and the accomplishment resides at the preferential choices level of the young people who constitute our 4th head of column in the framework of analysis. The fifth point is the achievement or realization of the preferential choice made. Schematically the framework described above is presented in Table 1 .

Table I Analytical frame of responsible sexuality through the capability approach

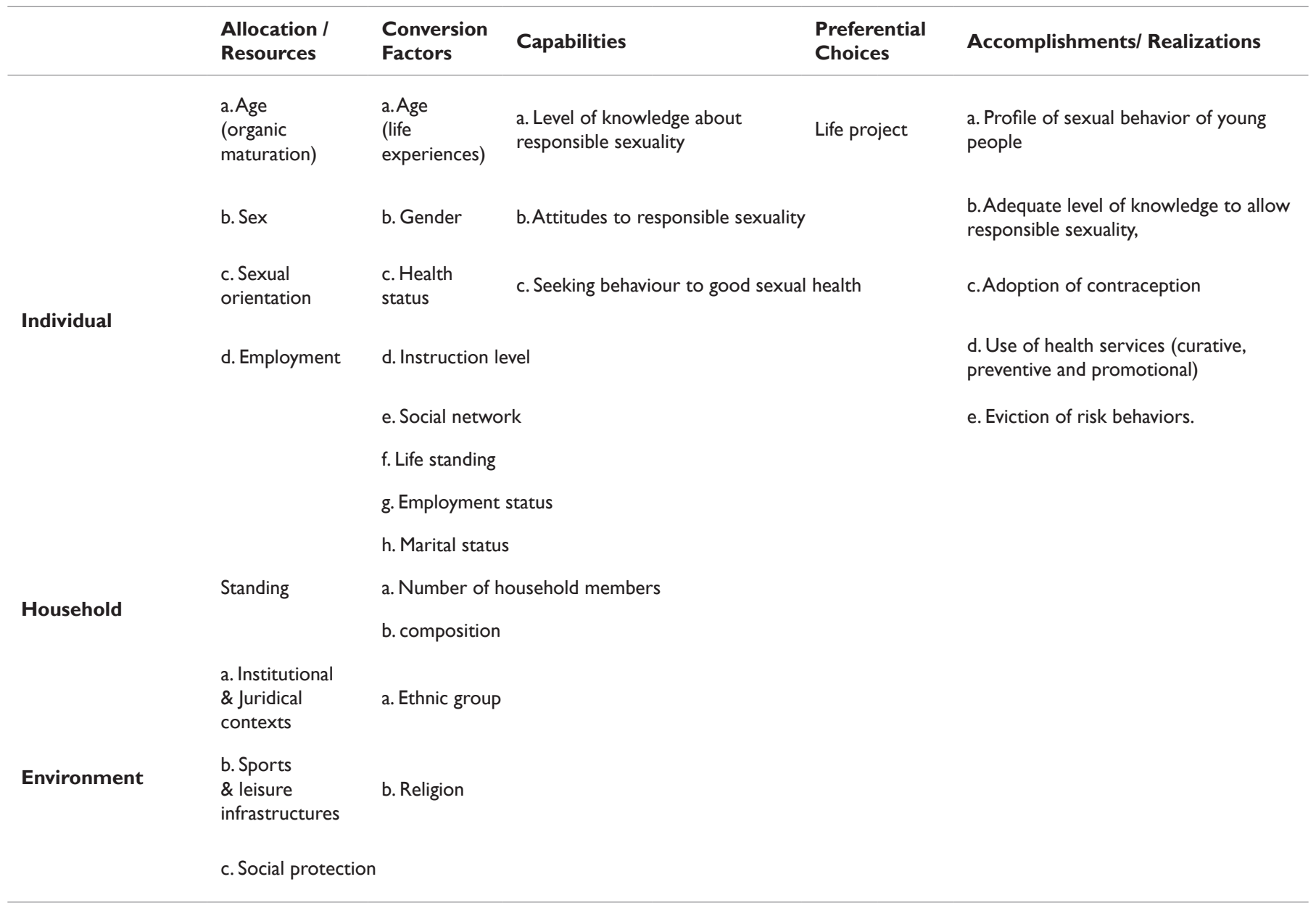


Box I
A young person, in the context of this study, is considered to be sexually responsible if he/she meets the following criteria:
a. Primary prevention:To delay the entry of sexual activity into young people up to the legal age of majority.
b. Secondary prevention: By becoming sexually active, systematically protect against STI/HIV/AIDS and early pregnancy.
c. Tertiary prevention: Seek adequate care in case of infection and early pregnancy.
The avoidance of risky behaviors such as alcohol consumption and the use of harmful substances is a cross-cutting measure in all areas of prevention.

\section{Results}

To conceptualize the application of the Capability Approach to Youth Responsible Sexuality, we have conceded that the content given to this concept encompasses the possibility for the young person to be an actor in his life and to have the level of knowledge needed to make healthy choices (consciously acting by knowing the consequences of one's actions, thinking about emotional and physical issues with the risks related to STIs, pregnancy). More explicitly, young people need to develop useful skills to delay till 18 years (or as long as possible) the onset of sexual activity and the adoption of contraceptive methods, the use of health services (Preventive, curative and promotional) and prevention of risk behaviors among young people. Considering the adoption of a responsible sexuality by young people in this aspect as the accomplishment to be achieved in the Capability Approach; we will review the factors in terms of resources, endowments and individual qualities upstream of this process result, taking into account the conversion factors identified by Sen in his economic model: Development, Justice, Freedom (Section: The Well- Being: diversity and heterogeneity). ${ }^{19}$ In the specific case of promoting sexual and reproductive health, we can assume that all the factors that influence the quality of life of an individual can have an effect on his sexuality by defining it. And the expression of sexual and reproductive rights and their consideration by public authorities also change the environment. The modeling of the relations between the individual and his choices in a given environment can be conceptualized as follows for the present study in the light of the Capability Approach (Figure 1).
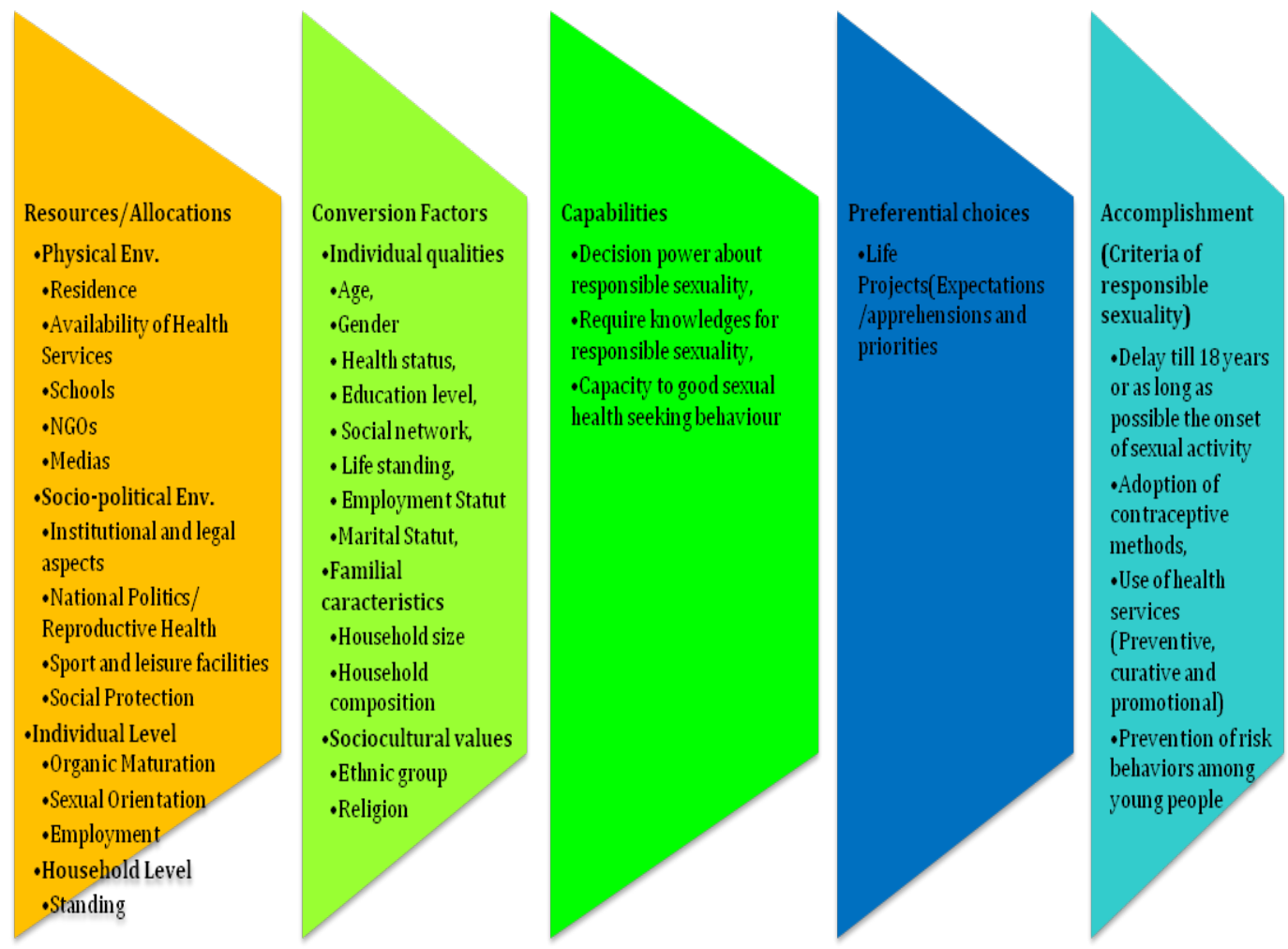

Figure I Conceptual framework of youth responsible sexuality the capability approach.

\section{Comments}

Sexual and reproductive rights issues and freedom issues about development are linked in a certain way because all of them have as their ultimate objective the quality of life and well-being of the individual. The analysis of responsible sexuality through the light of the Capability Approach seems to be fairly coherent under this auspice, especially for countries with low literacy rates and with strong socio- cultural pressures in our view. It will enable us to better understand the effect of cumbersomeness on the preferential choices of young people in terms of the expression of individual freedoms and specificities but also of norms and references. Indeed, in many African communities, girls often ask themselves whether they are really "women" because of the social considerations associated with this and to prove to them that they are, they will make choices in the realization of the answer to the question they are asking themselves. For promoters of sexual 
and reproductive health, young people must make informed decisions and not be subjected exclusively or indiscriminately to the weight of socio-cultural values and/or norms. This does not invite to the abandonment of a socio-cultural identity but to sort out what is to be maintained or promote from what is to be abandoned for the wellbeing of individuals. The conceptual framework we propose does not pretend to be perfect but with the Capability Approach as a tool we want to contribute to the already known fact that the emergence of peoples in terms of development probably related to questions of sexual and reproductive health through the preferential choices of young people. Since the principles of sexual and reproductive rights are universal and the Capability Approach has allowed for other dimensions of development to be taken into account than economic growth, the conceptual framework of responsible sexuality presented here can be exploited in all contexts.

\section{Conclusion}

The Capability Approach has been a topic of discussion for experts in many fields since the 2000s and its use in analyzing responsible sexuality of young people is understandable because of its contribution to the evaluation of development and individual's quality of life. The content of the concept of responsible sexuality has been defined and takes into account the useful skills that young people need to develop in order to delay as long as possible the initiation of sexual activity and the adoption of contraceptive methods, the use of reproductive health services (preventive, curative and promotional) and avoidance of risk behaviors. With the capability approach we have been able to identify the issues to be considered in the context of responsible sexuality of young people in order to propose a conceptual framework even though we admit that this same result can be achieved with other means. It took into account the levels of social structuring and the process necessary for the fulfillment of responsible sexuality among young people.

\section{Acknowledgements}

None.

\section{Conflict of interest}

Author declares that there is no conflict of interest.

\section{References}

1. Alkire S. The Capability Approach to the Quality of life. 2008.
2. Ingrid Robeyns. Capability Approach. SEP, Europe; 2016.

3. Sen AK. Well being Capability and Public policy. Annals of Econ. 1994;3(7-9):333-347.

4. Nussbaum M. Women and Human Development: The Capability Approach. USA: Cambridge University Press; 2000. p. 1-336.

5. Robeyns I. Sen's Capability approach and gender inequality: selecting relevant capabilities. Feminist Economics. 2003;9(2-3):61-92.

6. Solava SI. From Individual to Collective Capabilities: The Capability Approach as a Conceptual Framework for Self-help. Journal of Human Development. 2006;7(3):397-416.

7. Chiappero-Martinetti E, Moroni S. An analytical framework for conceptualizing poverty and re-examining the capability approach. The Journal of Socio-Economics. 2007;36(3):360-375.

8. Trani JF, Bakhshi P, Bellanca N, et al. Disabilities through the Capabilities Approach lens: Implications for public policies. European Journal of Disability Research. 2011;5(3):143-157.

9. UNDP. Human Development Report. New York: Oxford University Press; 1990. p. 1-141.

10. Mahbub Ul Haq. Reflections on Human Development. New York: Oxford University Press; 1995. p. 1-252.

11. UN. Report of the International Conference on Population and Development. New York, USA; 1994. p. 1-114.

12. Fathalla MF, Sinding SW, Rosenfield MM. Sexual and reproductive health for all: a call for action. The Lancet Sexual and Reproductive Health Series. 2006:1-6.

13. UN. Twenty-first special session of the General Assembly for an overall review and appraisal of the implementation of the POA of the ICPD. New York, USA; 1999:1-5.

14. AU. Maputo Protocol. Addis-Ababa, Africa; 2003.

15. AU. Maputo Plan of Action 2016-2030. Addis-Ababa, Africa; 2015:1-24.

16. Improving sexual and reproductive health. HIP. Africa; 2015.

17. Nussbaum M. Capabilities as fundamental entitlements: Sen and social justice. Feminist Economics. 2003;9(2-3):35-59.

18. Widdows $\mathrm{H}$, Law I. Conceptualising health: insights from the Capability Approach. Health Care Anal. 2007;16(4):303-314.

19. Sen A. A New Economic Model-Development, Justice, Freedom. Paris; 2003:1-5. 